\title{
Reorganização da atenção à saúde mental na pandemia de Covid-19
}

\author{
Reorganization of the Mental Health Care in the \\ Covid-19 pandemic
}

Flávia Caroline Figel ${ }^{1}$, Maristela da Costa Sousa ${ }^{2}$, Larissa Sayuri Yamaguchi ${ }^{3}$, Suelen Letícia Gonçalo ${ }^{4}$, Julia Eliane Murta ${ }^{5}$, Amanda Cristina Alves ${ }^{6}$

1. ORCID: https://orcid.org/0000-0001-9986-6813. Psicóloga. Mestrado. Secretaria de Estado da Saúde do Paraná, Curitiba, Paraná, Brasil. E-mail: flavia.figel@sesa.pr.gov.br.

2. ORCID: https://orcid.org/0000-0002-1653-8281. Médica Psiquiatra. Especialização. Secretaria de Estado da Saúde do Paraná, Curitiba, Paraná, Brasil. E-mail: maristelasousa@sesa.pr.gov.br.

3. ORCID: https://orcid.org/0000-0002-4577-3221. Psicóloga. Especialização. Secretaria de Estado da Saúde do Paraná, Curitiba, Paraná, Brasil. E-mail: larissa.yamaguchi@sesa.pr.gov.br.

4. ORCID: https://orcid.org/0000-0002-6145-4036. Assistente Social. Especialização. Secretaria de Estado da Saúde do Paraná, Curitiba, Paraná, Brasil. E-mail: suelen.goncalo@sesa.pr.gov.br.

5. ORCID: https://orcid.org/0000-0002-0492-6340. Psicóloga. Especialização. Secretaria de Estado da Saúde do Paraná, Curitiba, Paraná, Brasil. E-mail: julia.murta@sesa.pr.gov.br.

6. ORCID: https://orcid.org/0000-0002-1428-2657. Estagiária de Serviço Social. Graduanda. Secretaria de Estado da Saúde do Paraná, Curitiba, Paraná, Brasil. E-mail: amanda.cristina@sesa. pr.gov.br.

CONTATO: Flávia Caroline Figel | Endereço: Rua Piquiri, 170 - Rebouças - Curitiba-PR | Telefone: (41) 3330-4526 | E-mail: saudemental@sesa.pr.gov.br

RESUMO A pandemia por Covid-19 exigiu a reorganização dos serviços da saúde, inclusive da saúde mental. Este artigo é um relato de experiência, estruturado por meio de abordagem crítico reflexiva e descritiva, sobre as ações da Secretaria de Estado da Saúde do Paraná para favorecer a reorganização da Atenção à Saúde Mental diante do surto de coronavírus. Foi elaborada uma Nota Orientativa para possibilitar a manutenção do cuidado para usuários anteriormente atendidos pela Rede, assim como o acompanhamento daqueles que possam apresentar sofrimento mental em decorrência desse período de pandemia.

DESCRITORES: Saúde Mental. Saúde. Infecções por Coronavírus. Atenção à Saúde. 


\begin{abstract}
The pandemic of Covid-19 required the reorganization of health services, including the mental health. This article is an experience report, structured through a critical reflective and descriptive approach, about the actions of the Paraná State Department of Health to favor the reorganization of the Mental Health Care related to the coronavirus outbreak. It was elaborated a guidance note to enable the maintenance for users previously attended by the network, as well as tracking the users that can present mental suffering as a result of this pandemic period.
\end{abstract}

DESCRIPTORS: Mental Health. Health. Coronavirus Infections. Health Care.

\title{
INTRODUÇÃO
}

$\mathbf{A}$ pandemia da Covid-19 culminou na necessidade de reorganização da atenção à saúde, incluindo a saúde mental, intimamente relacionada ao impacto no cotidiano de vida e saúde. Considerando esse cenário desafiador, reflexões acerca dos obstáculos encontrados e das estratégias para superá-los têm sido publicadas. Algumas dessas estratégias envolvem a disponibilidade de equipes multiprofissionais de saúde mental, comunicação clara com atualizações regulares e acuradas sobre a epidemia, estabelecimento de serviços seguros para promover aconselhamento psicológico, tais como comunicação por meio de dispositivos eletrônicos e aplicativos'

No que concerne à Saúde Mental, diante do contexto da pandemia pelo SARS-CoV-2 é importante considerar que existem questões de saúde mental específicas desse período, e sofrimentos desencadeados pelas mudanças de vida decorrentes da insegurança nos aspectos de saúde, social e econômico. Neste especial período observa-se sentimentos comuns, tais como medos relativos às perdas da vida, de pessoas amadas e dos meios de subsistência. O medo também está associado à exclusão social frente à possível doença, ao isolamento, ao risco de ser a causa do adoecimento de outras pessoas, além da sensação recorrente de impotência perante os acontecimentos, irritabilidade, angústia e tristeza². 
Mediante o enfrentamento à pandemia no contexto da Atenção à Saúde Mental da Secretaria de Estado da Saúde do Paraná, este relato de experiência apresenta e discute as demandas e ações desenvolvidas com vistas a instrumentalizar as equipes de saúde que constituem a linha de frente no atendimento aos doentes e familiares, as quais também apresentam sofrimento mental neste momento único da saúde pública mundial.

\section{MÉTODO}

Trata-se de um relato de experiência, estruturado por meio da abordagem crítico reflexiva e descritiva, sobre as mudanças nos processos de trabalho da saúde mental e as ações realizadas pela Divisão de Atenção à Saúde Mental da Secretaria de Estado da Saúde do Paraná (SESA-PR) para favorecer a reorganização da Atenção à Saúde Mental diante do surto de coronavírus.

A construção do conteúdo foi estruturada na vivência da equipe multiprofissional da Divisão e normativas publicadas do período de fevereiro a julho de 2020, e ancorada em publicações relativas ao tema.

\section{RESULTADOS E DISCUSSÃO}

Desde antes da declaração de pandemia pela Organização Mundial da Saúde, mas frente aos inúmeros casos de Covid-19 relatados no mundo, se iniciou um processo de transformação, frente às constantes mudanças nas informações divulgadas, estudos científicos, diretrizes do Ministério da Saúde, da Fiocruz e demais instituições de saúde pública. Este contexto também contribuiu como fator ansiogênico porque trouxe insegurança, uma vez que inicialmente havia pouco conhecimento sobre o novo coronavírus, sua forma de propagação e contaminação, a fisiopatologia da Covid-19, sua morbimortalidade e os métodos mais eficazes de prevenção e tratamento. 
As reações do ser humano frente ao risco e ao desconhecido são múltiplas e decorrem de inúmeros fatores. Na atual situação de pandemia as intervenções devem focar não apenas na saúde física e no combate ao vírus, mas também na saúde mental da população³.

Considera-se que entre um terço e metade da população exposta a uma epidemia pode vir a sofrer alguma manifestação psicopatológica, caso não haja intervenção específica para as reações e sintomas manifestados². Fatores como a magnitude do evento e o grau de vulnerabilidade psicossocial dos indivíduos podem contribuir para o surgimento de algum transtorno psíquico ${ }^{4}$.

Ao se considerar a população em geral, que pode apresentar sofrimentos em decorrência da pandemia, e a fim de prevenir o agravamento de manifestações psicopatológicas, diversos municípios do Estado do Paraná instituíram acolhimento psicológico, por meio de acesso telefônico, a fim de oferecer apoio, inclusive direcionado a profissionais de saúde. Esses atendimentos são de grande importância, pois tem a capacidade de validar as reações da clientela, por meio da disponibilização de informações de prevenção e estratégias de enfrentamento em geral, sobretudo relativas ao manejo de estresse e ansiedade ${ }^{5}$.

Em pesquisa realizada na China, com 1.210 participantes de 194 cidades, foi identificado que $28,8 \%$ dos respondentes apresentou sintomas moderados a severos de ansiedade, enquanto $16,5 \%$ e $8,1 \%$ apresentaram sintomas de depressão e estresse, respectivamente 6 . Tendo isso em vista, ações de acolhimento também se mostram efetivas, considerando que a ansiedade pode fazer com que indivíduos interpretem sensações corporais de maneira equivocada, confundindo-as com doenças, o que contribui para que entendam ser necessário procurar os serviços de saúde, mesmo sem necessidade. Tais serviços também podem disponibilizar Primeiros Auxílios Psicológicos, que correspondem à intervenção precoce de saúde mental voltada às pessoas impactadas, de alguma forma, com os atuais eventos ${ }^{8-9}$. Sobremaneira, esses profissionais podem identificar os casos em que há necessidade do encaminhamento para o cuidado específico de saúde mental ${ }^{5}$. 
Sensível ao contexto da saúde mental frente à pandemia, a Divisão de Atenção à Saúde Mental publicou a Nota Orientativa $\mathrm{n}^{0} 15^{10} \mathrm{com}$ condutas em situações envolvendo a saúde mental e direcionadas aos serviços de saúde do Estado. O conteúdo foi elaborado em consonância com demais documentos orientativos em âmbito estadual e federal, com destaque à diretrizes do Ministério da Saúde ${ }^{11}$ e do Conselho Nacional de Secretários de Saúde ${ }^{12}$.

Mediante a necessidade de reorganização dos serviços de saúde, incluindo a Rede de Atenção Psicossocial, inúmeros processos foram desencadeados, desde os de suporte à prevenção da disseminação do vírus nos ambientes assistenciais, até o manejo diante das manifestações de sofrimento mental.

A reorganização referente aos aspectos sanitários, como disponibilização de álcool em gel e locais para higienização de mãos, recomendações para o distanciamento social, promoção da ventilação dos ambientes e limpeza após o uso, se deu como etapa elementar, porém demandou a compreensão de sua necessidade no contexto da saúde mental. Outros aspectos relativos a alterações nos processos de trabalho, para evitar aglomeração ou transmissão de possível contaminação entre as pessoas, ocorreram em toda a Rede de Atenção Psicossocial, com algumas diferenças de acordo com o nível de atenção. Nesse aspecto, priorizou-se a manutenção da assistência de modo que os usuários não ficassem desassistidos, o que poderia agravar ou agudizar suas condições de saúde. Também foi observado maior intercâmbio de estratégias de ações terapêuticas entre os profissionais, tanto na prática quanto nas discussões que ocorreram pelos meios virtuais.

É necessário considerar que pessoas com problemas de saúde mental podem apresentar respostas emocionais mais intensas frente à epidemia, resultando em maior suscetibilidade ao estresse, se comparados com a população geral ${ }^{13}$. Assim, esforços de prevenção, tais como o rastreio de problemas de saúde mental, psicoeducação e suporte psicossocial devem ser fornecidos e focados, especialmente, nos grupos mais vulneráveis nesse período de pandemia, que são as pessoas contaminadas pelo coronavírus, as do grupo de risco, além daquelas 
com doenças físicas e mentais preexistentes, ou que façam uso de substâncias psicoativas $^{5}$.

Na Atenção Primária à Saúde, no que tange à Saúde Mental, foi orientado que os serviços priorizassem formas alternativas de comunicação com os usuários, tais como, atendimento telefônico ou videochamadas. E que esses realizassem o acompanhamento mais próximo dos casos de usuários com maior risco de agravamento ou agudização dos sintomas, como, por exemplo, pessoas com transtornos psicóticos recém recuperados ou em fase aguda de tratamento, com transtornos depressivos que apresentem ideação suicida ou com abuso de álcool que necessite desintoxicação e abordagem medicamentosa, além daqueles usuários que apresentam maior vulnerabilidade social. Ademais, muitos municípios se organizaram para acompanhar, por telefone, usuários em quarentena; tal medida, além de favorecer o cuidado relativo à própria infecção, também auxilia no cuidado em saúde mental, pois permite a diminuição do impacto psicológico neste período ${ }^{14}$.

Na Atenção Especializada, os serviços ambulatoriais e os Centros de Atenção Psicossocial (CAPS) mantiveram as portas abertas, em sua maioria, sendo adotadas medidas para reduzir a circulação de pessoas do grupo de risco, evitar a aglomeração em atividades terapêuticas, priorizando atendimentos individuais e, de preferência, à distância, com a utilização de comunicação por meios eletrônicos.

Na Atenção Hospitalar foi elaborado Plano de Contingência e a Divisão de Saúde Mental realizou alterações em sua Nota Orientativa $n^{\circ} 15^{10}$ para contemplar as especificidades neste contexto assistencial e tentar minimizar possíveis dificuldades ante os desafios postos, especialmente após a ocorrência de um surto de Covid-19 em um dos hospitais do Estado. Portanto, houve a necessidade de criar áreas livres de Covid-19 para o atendimento dos usuários, adoção de isolamento e quarentena para casos suspeitos, bem como orientação da necessidade de avaliar para quais casos a hospitalização seria imprescindível, considerando os riscos neste período especial. Complementarmente, foi recomendado dar alta médica para pacientes hospitalizados estabilizados, com referenciamento para 
seguimento do tratamento tanto da atenção primária como especificamente em saúde mental. Também foram feitas orientações a pacientes e familiares quanto as rotinas de higienização, uso de máscaras, e circulação restrita. Medicações de uso contínuo foram dispensadas para maior intervalo de tempo, bem como os retornos para o monitoramento ambulatorial foram estendidos.

Nas mudanças dos processos de trabalho, os recursos tecnológicos são importantes ferramentas durante a pandemia, viabilizando o acesso dos profissionais de saúde aos usuários, permitindo a realização de monitoramento e, até mesmo, de alguns atendimentos, como no caso da saúde mental ${ }^{15}$. A utilização desses recursos mostrou vantagens e desvantagens. As vantagens se referem à possibilidade de manter o contato com os usuários, apesar do distanciamento social e isolamento, e garantir que aqueles em atendimento não presencial sejam acompanhados em suas necessidades. Entretanto, algumas desvantagens dizem respeito às dificuldades de acesso de usuários que não dispõem de conexão de internet satisfatória, ou de telefone. Além disso, alguns usuários têm baixa adesão a essas modalidades, muitas vezes por apresentarem dificuldades no uso desses equipamentos, ou em virtude da ausência de privacidade necessária na residência, que não permite falar abertamente sobre questões sigilosas ou de foro íntimo. Há também questões culturais, como viver em área rural e/ ou não valorizar o uso de dispositivos tecnológicos, contribuindo para que esses usuários apresentem mais resistência a utilizar a tecnologia para atendimento de saúde, além de questões específicas de faixas etárias, como entre idosos. Inclusive, entre essa população pode haver a necessidade de planejamento de intervenções que contemplem suas especifidades ${ }^{16}$.

Tais vivências são, contudo, de suma importância para que possam ser construídas estratégias eficazes de atendimento em saúde mental, que garantam o acesso de maior parcela da população. Diminuir as barreiras de acesso se faz necessário não apenas no período de pandemia, pois a garantia do cuidado em saúde mental é fator protetivo para diversas psicopatologias. Inclusive, pode prevenir o suicídio, tendo em vista que alguns fatores constituem risco para o 
suicídio e estão presentes durante essa pandemia, tais como agravamento de transtornos mentais, desemprego, dificuldades financeiras, violência doméstica, aumento do uso de álcool, isolamento social e solidão ${ }^{17-18}$, evidenciando a necessidade de serem planejadas ações de prevenção.

Com relação aos profissionais da saúde, é necessário considerar que esses estão ainda mais expostos a estressores relacionados à pandemia e podem apresentar exaustão emocional, irritabilidade, mudanças nos hábitos alimentares e dificuldades para dormir ${ }^{19}$. Também esses podem apresentar mais sintomas psicológicos ao precisarem fazer a quarentena ${ }^{14}$. Disponibilizar o acolhimento psicológico é importante estratégia de cuidado em saúde mental, embora em muitos casos ocorra baixa adesão a esses serviços, talvez relacionada à grande sobrecarga de trabalho e falta de tempo ${ }^{20}$ ou então à preocupação com outras questões, considerando as intervenções psicológicas como secundárias ${ }^{21}$.

Importante desafio é garantir que as ações de saúde mental façam parte do cuidado geral de saúde neste período de pandemia ${ }^{5}$, pois a desassistência concorre para o aumento das demandas de saúde mental na pós-pandemia. Alguns estudos já identificaram quais seriam os grupos mais vulneráveis nesse período: crianças e adolescentes, idosos, pessoas em situação de rua, pessoas diagnosticadas ou com suspeita de Covid-19, profissionais de saúde que atuam na linha de frente, pessoas com transtornos mentais ou que façam uso de alguma substância psicoativa ${ }^{22}$. Assim, é necessário pensar em estratégias para lidar com os problemas que possam surgir em decorrência da atual pandemia.

Embora o isolamento social recomendado seja amenizado pelo uso de meios de comunicação virtual, há a necessidade do contato físico entre as pessoas como forma de diminuir a angústia mobilizada pelo medo das perdas e pelo isolamento social prolongado. Tendo em vista que o ser humano necessita das manifestações de carinho, amor, aceitação e afeto presencial para fortalecer a noção de indivíduo, obter provas de reconhecimento e da própria existência no mundo, o tempo prolongado de isolamento pode esgotar as capacidades de resiliência necessárias para sua adaptação ao "novo normal". Portanto, algo que precisa ser 
considerado pelos trabalhadores da área da saúde é que, sem desconsiderar o risco de contaminação, práticas alternativas precisam ser criadas para amenizar o sofrimento e o estresse causados pela vivência da opressão causada pela ameaça que representa a pandemia na vida das pessoas.

É preciso ponderar que como evento inesperado, a pandemia impossibilitou o planejamento de ações mais efetivas e muito do que tem sido realizado globalmente precisou ser executado num curto período de tempo. Portanto, as reflexões realizadas a partir do atual contexto devem servir de aprendizado para futuras epidemias ou pandemias ${ }^{23}$.

\section{CONSIDERAÇÕES FINAIS}

Tendo em vista esse momento único da Saúde Pública, se faz necessária a construção de novas estratégias para lidar com os desafios decorrentes da pandemia por Covid-19. É preciso desenvolver ações alternativas que permitam a promoção da saúde mental e a prevenção de problemas potenciais. Esse contexto implica que profissionais se reinventem e descubram novas formas de buscar aproximação com os usuários, formas de preservar ou mesmo criar vínculos terapêuticos. O adoecimento mental progressivo é testemunhado pela população, vulnerável ou não, na medida em que a pandemia persiste e determina progressiva hospitalização, isolamento e óbito de pessoas, próximas ou não, gerando insegurança.

Esse novo lugar da saúde mental a evidencia como área transversal e que acompanha a vida de todos em todos os momentos de sua existência. Assim, a promoção e a prevenção em saúde mental, não apenas o tratamento dos agravos, são essenciais. Ações diversas, com destaque ao acolhimento, proporcionado pelos profissionais de saúde, bem amparados por seu capital emocional e pela própria bagagem de conhecimento técnico-científico, são de grande valia para reduzir o impacto da pandemia sobre a saúde física e mental. 


\title{
AGRADECIMENTOS
}

\author{
Agradecemos a colaboração da Dra. Elaine Drehmer de Almeida Cruz na \\ elaboração do texto.
}

\section{REFERÊNCIAS}

1. Park S, Park YC. Mental Health Care Measures in Response to the 2019 Novel Coronavirus Outbreak in Korea [Internet]. J Korean Neuropsychiatr Assoc; 2020. [citado em 2020 Jul 31]; 17(2): 85-86. Disponível em: http://dx.doi.org/10.30773/ pi.2020.0058

2. Noal DS, Damásio F, Melo BD, Pereira DR, Serpeloni F, Kabad JF, et al. Saúde Mental e Atenção Psicossocial na Pandemia COVID-19: Recomendações Gerais [Internet]. Rio de Janeiro: Fiocruz; 2020a. [citado em 2020 Jul 31]. Disponível em: https:// www.fiocruzbrasilia.fiocruz.br/wp-content/uploads/2020/04/Sa\%C3\%BAde-Mental-e-Aten\%C3\%A7\%C3\%A3o-Psicossocialna-Pandemia-Covid-19-recomenda\%C3\%A7\%C3\%B5es-gerais.pdf.

3. Borloti E, Haydu VB, Kienen N, Zacarin MRJ. Saúde Mental e Intervenções Psicológicas durante a pandemia da COVID-19: um panorama [Internet]. Rev. bras. anál comport; 2020 [citado em 2020 Jul 31]; 16(1): 21-30. Disponível em: http://dx.doi. org/10.18542/rebac.v16i1.8885.

4. Noal DS, Damásio F, Melo BD, Pereira DR, Serpeloni F, Kabad JF, et al. Saúde Mental e Atenção Psicossocial na Pandemia COVID-19: Recomendações para Gestores [Internet]. Rio de Janeiro: Fiocruz; 2020b. [citado em 2020 Jul 31]. Disponível em: https://www.fiocruzbrasilia.fiocruz.br/wp-content/uploads/2020/04/Sa\%c3\%bade-Mental-e-Aten\%c3\%a7\%c3\%a3oPsicossocial-na-Pandemia-Covid-19-recomenda\%c3\%a7\%c3\%b5es-para-gestores.pdf.

5. Pfefferbaum B, North CS. Mental Health and the Covid-19 Pandemic [Internet]. N EngI J Med.; 2020. [citado em 2020 Jul 31]; 383: 510-512. Disponível em: https://www.nejm.org/doi/full/10.1056/NEJMp2008017.

6. Wang C, Pan P, Wan X, Tan Y, Xu L, Ho CS, et al. Immediate psychological responses and associated factors during the initial stage of the 2019 coronavirus disease (COVID-19) epidemic among the general population in China [Internet]. Int J Env Res Pub He; 2020. [citado em 2020 Jul 31]; 17(5): 1729. Disponível em: https://doi.org/10.3390/ijerph17051729.

7. Asmundson GJG, Taylor S. Coronaphobia: Fear and the 2019-nCov Outbreak [Internet]. J. Anxiety Disord; 2020. [citado em 2020 Jul 31]; 70: 102196. Disponível em: https://doi.org/10.1016/j.janxdis.2020.102196.

8. Everly GSJr, Flynn BW. Principles and practical procedures for acute psychological first aid training for personnel without mental health experience [Internet]. Int. J. Emerg. Ment. Health; 2006. [citado em 2020 Jul 31]; 8(2): 93-100. Disponível em: https://psycnet.apa.org/record/2006-06513-003.

9. Shah K, Kamrai D, Mekala H, Mann B, Desai K, Patel RS. Focus on mental health during the coronavirus (COVID-19) pandemic: applying learnings from the past outbreaks [Internet]. Cureus; 2020. [citado em 2020 Jul 31]; 12(3): e7405. Disponível em: https://dx.doi.org/10.7759\%2Fcureus.7405.

10. Secretaria de Estado da Saúde do Paraná. Nota Orientativa nº 15: Saúde Mental. [Internet]. Paraná: Secretaria de Estado da Saúde do Paraná; 2020. [citado em 2020 Jul 31]. Disponível em: https://www.saude.pr.gov.br/sites/default/arquivos restritos/files/documento/2020-06/no_15_saude_mental_v2.pdf.

11. Ministério da Saúde. Nota Técnica no 12/2020-CGMAD/DAPES/SAPS/MS [Internet]. Brasília: Ministério da Saúde; 2020a. [citado em 2020 Jul 31]. Disponível em: http://189.28.128.100/dab/docs/portaldab/documentos/ notatecnica122020CGMADDAPESSAPSMS02abr2020COVID-19.pdf.

12. Conselho Nacional de Secretarias Municipais de Saúde e Conselho Nacional de Secretários de Saúde. Guia Orientador para o enfrentamento da pandemia Covid-19 na Rede de Atenção à Saúde [Internet]. Brasília: Conselho Nacional de Secretarias Municipais de Saúde e Conselho Nacional de Secretários de Saúde; 2020b. [citado em 2020 Jul 31]. Disponível em: https:// www.conasems.org.br/wp-content/uploads/2020/05/Instrumento-Orientador-Conass-Conasems-VERS\%C3\%830-FINAL-3. pdf.

13. Yao H, Chen H, Xu Y. Patients with mental health disorders in the COVID-19 epidemic [Internet]. Lancet; 2020. [citado em 2020 Jul 31]; 7(4): e21. Disponível em: https://doi.org/10.1016/S2215-0366(20)30090-0.

14. Brooks SK, Webster RK, Smith LE, Woodland L, Wessely S, Geenberg N, et al. The psychological impact of quarantine and 
how to reduce it: rapid review of evidence [Internet]. Lancet; 2020. [citado em 2020 Jul 31]; 395: 912-920. Disponível em: https://doi.org/10.1016/S0140-6736(20)30460-8.

15. Liu S, Yang L, Zhang C, Xiang Y, Liu Z, Hu S, et al. Online mental health services in China during the COVID-19 outbreak [Internet]. Lancet; 2020. [citado em 2020 Jul 31]; 7(4): e17-e18. Disponível em: https://doi.org/10.1016/S2215-0366(20)300778.

16. Yang Y, Li W, Zhang Q, Zhang L, Cheung T, Xiang Y. Mental health services for older adults in China during the COVID-19 outbreak [Internet]. Lancet; 2020. [citado em 2020 Jul 31]; 7(4): e19. Disponível em: https://doi.org/10.1016/S22150366(20)30079-1.

17. Gunnell D, Appleby L, Arensman E, Hawton K, John A, Kapur N, Khan M, O'Connor RC, Pirkis J. Suicide risk and prevention during the COVID-19 pandemic [Internet]. Lancet Psychiat; 2020. [citado em 2020 Jul 31]; 7(6): 468-471. Disponível em: https://doi.org/10.1016/S2215-0366(20)30171-1.

18. Reger MA, Stanley IH, Joiner TE. Suicide mortality and coronavirus disease 2019 - A perfect storm? [Internet] JAMA Psychiatry; 2020. [citado em 2020 Jul 31]. Disponível em: http://dx.doi.org/10.1001/jamapsychiatry.2020.1060.

19. Barello S, Palamenghi L, Graffigna G. Burnout and somatic symptoms among frontline healthcare professionals at the peak of the Italian COVID-19 pandemic [Internet]. Psychiatry Res; 2020. [citado em 2020 Jul 31]; 290: 113129. Disponível em: https://doi.org/10.1016/j.psychres.2020.113129.

20. Li W, Yang Y, Liu Z, Zhao Y, Zhang Q, Zhang L, et al. Progression of Mental Health services during the COVID-19 outbreak in China [Internet]. Int J Biol Sci; 2020. [citado em 2020 Jul 31]; 16(10): 1732-1738. Disponível em: http://dx.doi.org/10.7150/ ijbs.45120.

21. Schmidt B, Crepaldi MA, Bolze SDA, Neiva-Silva L, Demenech LM. Saúde mental e intervenções psicológicas diante da pandemia do novo coronavírus (COVID-19) [Internet]. Estud. Psicol; 2020. [citado em 2020 Jul 31]; 37. Disponível em: http:// dx.doi.org/10.1590/1982-0275202037e200063.

22. Das N. Psychiatrist in post-COVID-19 era - are we prepared? [Internet] Asian J Psychiatr; 2020. [citado em 2020 Jul 31]; 51: 1-2. Disponível em: http://dx.doi.org/10.1016/j.ajp.2020.102082.

23. Holmes EA, O'Connor RC, Perry H, Tracey I, Wessely S, Arseneault L, et al. Multidisciplinary research priorities for the Covid-19 pandemic: a call for action for mental health science [Internet]. Lancet; 2020. [citado em 2020 Jul 31]; 7(6): 547-560. Disponível em: https://doi.org/10.1016/S2215-0366(20)30168-1.

\section{RECEBIDO: 10/08/2020 \\ ACEITO: 15/10/2020}

